\title{
ERRATUM
}

\section{Mutation analysis of the $G N E$ gene in Korean patients with distal myopathy with rimmed vacuoles}

\author{
Byoung Joon Kim · Chang-Seok Ki $\cdot$ \\ Jong-Won Kim · Duk Hyun Sung · Young-Chul Choi · \\ Seung Hyun Kim
}

Published online: 30 August 2006

(C) The Japan Society of Human Genetics and Springer 2006

\section{Erratum to: J Hum Genet 51(2):137-140 DOI 10.1007/s10038-005-0338-5}

In this article, one of the novel mutations, A591T, was incorrectly given as A591P. In all occurrences throughout the article, the correct nomenclature of the novel mutation should be A591T.

The online version of the original article can be found at http://dx.doi.org/10.1007/s10038-005-0338-5.

B. J. Kim

Department of Neurology, Samsung Medical Center,

Sungkyunkwan University School of Medicine,

Seoul, South Korea

C.-S. Ki $(\bowtie) \cdot$ J.-W. Kim

Department of Laboratory Medicine,

Samsung Medical Center,

Sungkyunkwan University School of Medicine,

Seoul, South Korea

e-mail: changski@skku.edu

D. H. Sung

Department of Physical Medicine and Rehabilitation,

Samsung Medical Center,

Sungkyunkwan University School of Medicine,

Seoul, South Korea

Y.-C. Choi $(\square)$

Department of Neurology,

Brain Korea 21 Project for Medicine,

Yonsei University College of Medicine,

Seoul, South Korea

e-mail: ycchoi@yumc.yonsei.ac.kr

\section{S. H. Kim}

Department of Neurology, College of Medicine,

Hanyang University, Seoul, South Korea 